\title{
A Inclusão no Trabalho sob a Perspectiva das Pessoas com Deficiência Intelectual
}

\author{
Nara Liana Pereira-Silva ${ }^{1, *}$ \\ Orcid.org/0000-0002-8435-3078 \\ Adelaine Vianna Furtado ${ }^{2}$ \\ Orcid.org/0000-0003-3352-3812 \\ Jaqueline Ferreira Condé de Melo Andrade ${ }^{1}$ \\ Orcid.org/0000-0002-1303-2867
}

${ }^{1}$ Universidade Federal de Juiz de Fora, Juiz de Fora, MG, Brasil

${ }^{2}$ Centro de Referência de Assistência Social da Prefeitura Municipal de Muriaé, MG, Brasil

\section{Resumo}

A inclusão no mercado de trabalho representa, para as pessoas com deficiência intelectual, a possibilidade de desenvolvimento social e emocional. O presente estudo teve por objetivo investigar aspectos relacionados à inclusão no trabalho de pessoas com deficiência intelectual, a partir de relatos de experiências. Participaram quatro mulheres e cinco homens com idades entre 23 a 40 anos e diagnóstico de deficiência intelectual. Todos os participantes já possuíam experiência de trabalho, sendo que quatro estavam empregados e cinco estavam desempregados. Para a coleta de dados, os participantes foram visitados em suas residências, tendo respondido a um questionário e a entrevistas semiestruturadas. Os resultados das entrevistas demonstraram que a inclusão no mercado de trabalho é um processo mais satisfatório do que insatisfatório, além de ser um direito. Os dados apontam, portanto, que este contexto pode favorecer o desenvolvimento da autonomia, independência, satisfação pessoal, produtividade e responsabilidade. Ressalta-se a importância do planejamento de estudos que visem aprofundar o processo de inclusão no trabalho, destacando seu papel para o desenvolvimento das pessoas com deficiência intelectual, fornecendo dados empíricos que possam subsidiar a implementação e o planejamento de políticas públicas de inclusão.

Palavras-chave: Deficiência intelectual, mercado de trabalho, inclusão.

\section{Workplace Inclusion from the Standpoint of Individuals with Intellectual Disabilities}

\begin{abstract}
Inclusion in the job market offers intellectually disabled individuals the possibility of social and emotional development. The present study sought to explore several aspects of the workplace inclusion

* Endereço para correspondência: Rua José Lourenço Kelmer, s/n, Martelos, Juiz de Fora, MG, Brasil 36036330. E-mail: naraliana.silva@ufjf.edu.br

Apoio financeiro: Coordenação de Aperfeiçoamento de Pessoal de Nível Superior (Capes)

Nota do autor: este trabalho é parte da dissertação de mestrado defendida pela segunda autora com orientação da primeira.
\end{abstract}


process of individuals with intellectual disabilities, based on their accounts of personal experiences. The study enjoyed the participation of four women and five men between 23 and 40 years of age and diagnosed with intellectual disabilities. All of the participants had prior employment experience; four were employed at the time, and five were unemployed. In order to collect the research data, the participants were visited at their homes, where they answered a questionnaire and a semi-structured interview. The results of the interviews revealed that the workplace inclusion process is largely satisfactory and that inclusion is a right. The results thus indicate that the inclusion context is capable of aiding the development of autonomy, independence, personal satisfaction, productivity and responsibility. We stress the importance of preparing studies aimed at improving the workplace inclusion process, highlighting its role in the development of people with intellectual disabilities by providing empirical data that can support the planning and implementation of public policies on workplace inclusion.

Keywords: Intellectual disability, labor market, inclusion.

\section{La Inclusión en el Trabajo desde la Perspectiva de las Personas con Discapacidad Intelectual}

\section{Resumen}

La inclusión en el mercado de trabajo representa para las personas con discapacidad intelectual la posibilidad de desarrollo social y emocional. El presente estudio tuvo como objetivo investigar algunos aspectos de la inclusión laboral de personas con discapacidad intelectual a partir de relatos de experiencia. Participaron del estudio cuatro mujeres y cinco hombres con edades entre los 23 a 40 años que han sido diagnosticados con deficiencia intelectual. Todos los participantes ya tenían algún tipo de experiencia laboral; cuatro de ellos estaban trabajando, mientras que los otros cinco se encontraban desempleados. Para la recolección de datos los participantes fueron visitados en sus residencias respondiendo a un cuestionario y a entrevistas semiestructuradas. Los resultados de las entrevistas demostraron que la inclusión en el mercado de trabajo es un proceso más satisfactorio que insatisfactorio. Por tanto, los datos señalan que este contexto puede favorecer el desarrollo de la autonomía, independencia, satisfacción personal, productividad y responsabilidad. Es de resaltar la importancia de futuras investigaciones que profundicen en la inclusión laboral, destacando su papel para el desarrollo de personas con discapacidad intelectual proporcionado datos empíricos que puedan sustentar el diseño y la implementación de políticas públicas para la inclusión.

Palabras clave: Deficiencia intelectual, mercado de trabajo, inclusión.

Diversos foram os paradigmas existentes na história que influenciaram a construção de leis e o modo como as pessoas com deficiência foram e continuam sendo tratadas na vida em sociedade. Atualmente, o modelo da inclusão vem influenciando as políticas de atenção às pessoas com deficiência (Araújo, Escobal, \& Ribeiro, 2006; Mantoan, 2015; Mendes, 2006; Paiva, Bendassolli, \& Torres, 2015; Sassaki, 1997). De acordo com este paradigma, a deficiência não está instalada no indivíduo, cabendo somente a ele buscar o seu desenvolvimento, mas a sociedade também possui o papel de encontrar meios que proporcionem o desenvolvimento e a inclusão das pessoas com deficiência, tendo em vista o direito destas ao acesso a bens comuns da vida em comunidade (Aranha, 2001; Barbosa-Gomes \& Carvalho, 2010; Mantoan, 2015). Assim, na elaboração de políticas públicas, legislações e serviços direcionados às pessoas com deficiência intelectual (DI) têm sido centrais os conceitos como inclusão social, participação na comunidade e empoderamento (Morin, Rivard, Crocker, Boursier, \& Caron, 2013).

Nessa direção, o mercado de trabalho é um dos contextos de maior relevância para os adul- 
tos com DI, uma vez que este se apresenta como um ambiente onde esses adultos podem exercer sua cidadania, além de desenvolver habilidades sociais, cognitivas e relacionais. Historicamente, o acesso da pessoa com deficiência ao mercado de trabalho tem sido garantido por dispositivos legais. No âmbito nacional, desde a Constituição Brasileira de 1988 (artigo 37, VIII) se identifica a inclusão das pessoas com deficiência em vagas de emprego, especialmente, quando esta descreve sobre a reserva percentual de cargos em empregos públicos para estas pessoas. Em 1990 este direito foi reassegurado pela lei federal $\mathrm{n}^{\circ}$ 8.112 que, em seu artigo quinto, propôs a reserva de um percentual dos cargos públicos às pessoas com deficiência e definiu os critérios para essa admissão (Lei Federal ${ }^{\circ}$ 8.112, 1990). Em 1991, a Lei $\mathrm{n}^{\circ} 8.213$, conhecida como Lei de Cotas, mudou significativamente a forma de inserção dessa população nas organizações do trabalho (Cezar, 2010; Lobato, 2009). Essa lei dispõe sobre planos e benefícios da Previdência Social e estabelece, por meio de uma ação afirmativa, a reserva legal de cargos para pessoas com deficiência, obrigando as empresas, a partir de cem (100) funcionários, a preencherem uma parcela que varia de 2 a 5\% de seus cargos com pessoas com alguma deficiência (Lei $\left.n^{\circ} 8.213,1991\right)$. Em 2016 entrou em vigor a 2015, também conhecida como Lei Brasileira de Inclusão (Lei 13.146, 2015). Essa normativa assegura às pessoas com deficiências, dentre outros, "o direito ao trabalho de sua livre escolha e aceitação, em ambiente acessível e inclusivo, em igualdade de oportunidades com as demais pessoas". Essa lei avança na garantia de importantes direitos não apenas no âmbito do trabalho, mas na educação, acessibilidade e no combate ao preconceito e à discriminação. Internacionalmente, desde 1946, a Declaração Universal dos Direitos Humanos já garantia o "direito ao trabalho, à livre escolha de emprego, a condições justas e favoráveis de trabalho e à proteção contra o desemprego" (Organização das Nações Unidas [OMS], 1948, p. 12) a qualquer pessoa, concebendo o emprego como uma necessidade e um direito humano.

Entretanto, apesar do movimento em torno da inclusão e das garantias legais ofereci- das, poucas são as pessoas com DI inseridas no mercado de trabalho e, entre aquelas inseridas, muitas são as dificuldades enfrentadas para a sua permanência no emprego (Almeida, Carvalho-Freitas, \& Marques, 2010; Furtado \& Pereira-Silva, 2014; Lobato, 2009; Paiva et al., 2015). No Brasil, os dados obtidos no censo de 2010 apontam a existência de 45,6 milhões de pessoas com deficiência, representando $23,9 \%$ da população. Deste total, daqueles em idade para trabalhar, apenas, 46,2\% exercem alguma atividade ocupacional. As pessoas com DI representam $1,4 \%$ da população geral de pessoas com deficiência e daqueles em idade para trabalhar, apenas, $20,2 \%$ dos homens e $14,2 \%$ das mulheres trabalham. As pessoas com DI são, portanto, as menos absorvidas pelo mercado de trabalho, comparadas com as pessoas com outras deficiências, tais como a auditiva, a física e a visual (Instituto Brasileiro de Geografia e Estatística [IBGE], 2012). Ressalta-se, como fatores para este cenário, o descumprimento dos atributos legais, a carência de programas de formação profissional, a falta de acessibilidade física e social nas empresas, além do preconceito em relação à contratação da pessoa com deficiência intelectual (Araújo \& Schmidt, 2006; Lobato, 2009; Masson, 2009; Nord, Luecking, Mank, Kiernan, \& Wray, 2013; Nota, Santilli, Ginevra, \& Soresi, 2014; Tanaka \& Manzini, 2005; Vornholt, Uitdewilligen, \& Nijhuis, 2013).

Apesar dos desafios existentes, é fundamental a busca de ações que efetivem a inclusão da pessoa com DI no mercado de trabalho, uma vez que o ambiente de trabalho se constituir em um contexto de desenvolvimento, propiciando (ou não) a construção da identidade e de habilidades, a satisfação de necessidades básicas e de objetivos pessoais, a participação no desenvolvimento social e econômico da comunidade e o sentimento de valorização pessoal (Gold, Fabian, \& Luecking, 2013; Kirsh et al., 2009; Lysaght, Cobigo, \& Hamilton, 2012; Lysaght, Petner-Arrey, Cobigo, \& Oullette-Kuntz, 2014; Nord et al., 2013; Nota et al., 2014; Vornholt et al., 2013). Além disso, as pessoas com DI podem ter aumentadas suas redes de convívio, estabelecendo relações sociais e profissionais com pessoas sem deficiên- 
cia (Nota et al., 2014; Lysaght et al., 2012; Vornholt et al., 2013), reduzindo o isolamento social (Vieira \& Denari, 2008).

Segundo Akkerman, Janssen, Kef e Meininger (2014) e Pereira-Silva e Furtado (2012), uma das ações fundamentais para o provimento de suporte adequado e para a redução de barreiras à entrada no mercado de trabalho reside na investigação do que as próprias pessoas com DI consideram importante para sua satisfação no trabalho. Pereira-Silva e Furtado investigaram o processo de inclusão de quatro aprendizes com DI com idades entre 19 e 32 anos que possuíam experiência ocupacional no comércio. Os resultados das entrevistas evidenciaram que a falta de preparação profissional, as questões pessoais e socioemocionais dos aprendizes, bem como o despreparo das empresas em receber o trabalhador com DI eram os fatores mais responsáveis pelo insucesso da inclusão no mercado de trabalho. As autoras ressaltam a importância da interlocução entre instituições profissionalizantes e as empresas que recebem esses trabalhadores.

Akkerman et al. (2014) investigaram a perspectiva de nove pessoas com DI sobre a satisfação no trabalho. Como técnica de coleta de dados foi utilizada photovoice, a qual consistiu em que os participantes tirassem fotos do ambiente de trabalho e depois, por meio de uma entrevista, relatassem os seus registros fotográficos. A análise das entrevistas derivou nove temáticas, tendo cada uma delas condição de afetar positiva ou negativamente a percepção da satisfação com o trabalho. O tipo de trabalho e as condições nas quais ele é desempenhado, bem como as relações sociais estabelecidas no emprego foram amplamente apontadas pelos participantes como condições relevantes para sua satisfação naquele ambiente. Também foi mencionada a importância do suporte recebido e das oportunidades para o uso de competências. Ainda que em menor frequência, segundo os investigadores talvez por serem de mais difícil expressão, os trabalhadores com DI apontaram para as demandas de trabalho experienciadas, a autonomia percebida, oportunidades para o crescimento/desenvolvimento e a significância do trabalho.
A literatura tem mostrado que as pessoas com DI encontram dificuldades tanto para serem empregadas como para se manterem em seus empregos. Essa questão orientou o estudo de Holwerda, van der Klink, de Boer, Groothoff e Brouwer (2013) que, através da utilização de um follow-up, investigaram as variáveis que têm influência no comportamento de conseguir e manter o emprego. Participaram da pesquisa 735 adultos neerlandeses com DI com idades entre 15 a 27 anos. A motivação, as expectativas em relação à progressão no emprego, bem como a situação de moradia foram as principais variáveis preditivas de conseguir e manter-se em um emprego. Segundo os autores, quando o adulto tem motivação e expectativas positivas quanto à sua capacidade de trabalhar, ele se vê estimulado a buscar emprego ou a se manter naquele em que está contratado. Quanto à situação de moradia, aqueles participantes que viviam com a família ou independentemente obtiveram mais sucesso na busca e na manutenção do emprego do que os que viviam em instituições. Os autores concluíram que é fundamental que pais, professores e outros profissionais encorajem os adultos com DI a expressarem seus desejos, expectativas e interesses em relação ao mundo do trabalho, bem como ofereçam ajuda no desenvolvimento de expectativas realistas, propiciando, também, um ambiente de trabalho no qual haja constante suporte para que a pessoa com DI mantenha-se empregada.

Destaca-se a importância do trabalho como possibilidade de obtenção de satisfação com a vida e, consequentemente, melhor qualidade de vida dos adultos com DI. Nesse sentido, Santilli, Nota, Ginevra e Soresi (2014) investigaram 120 trabalhadores italianos com DI em relação à satisfação com a vida associando com a adaptabilidade à carreira e esperança. Foram encontradas relações entre essas duas variáveis, indicando haver associação entre a determinação para iniciar e sustentar comportamentos que visam alcançar metas e os procedimentos/meios para alcançar objetivos, o que conduz a mais satisfação com a vida. Segundo os autores, esses resultados indicam a importância de atividades que 
promovam a adaptação à carreira, favorecendo o crescimento motivacional e a qualidade de vida no ambiente de trabalho. Além disso, os autores destacam o importante papel dos profissionais que trabalham com essas pessoas, os quais devem encorajá-las e promover ações/intervenções que propiciem o planejamento de objetivos futuros, tendo como foco suas potencialidades e não as suas deficiências e dificuldades.

Não há dúvidas, portanto, sobre a importância do ambiente de trabalho para as pessoas com DI. Essa é uma área de pesquisa em expansão e não consolidada, conforme apontam os resultados de Furtado e Pereira-Silva (2014), carecendo de mais estudos. Dessa forma, a presente pesquisa, buscando responder ao seguinte questionamento "Como tem sido ou foram as experiências laborais de pessoas com DI?", teve o objetivo geral de investigar aspectos relacionados ao processo de inclusão de pessoas com DI no mercado de trabalho, a partir de relatos de suas experiências, focalizando: (a) o período de escolarização, (b) o significado de trabalhar, (c) a percepção sobre o ambiente de trabalho e as relações interpessoais, (d) o papel da família na inclusão, (e) as realizações com o trabalho, (f) as expectativas em relação ao emprego.

\section{Método}

\section{Participantes}

A presente pesquisa contou com a participação de nove pessoas adultas com o diagnóstico de DI. Os critérios de inclusão envolveram: (a) apresentar diagnóstico de DI, sem comorbidades, tendo sido emitido por um médico ou através de relatório psicossocial/psicopedagógico; (b) possuir idade mínima de 18 anos; (c) residir com os genitores ou responsáveis, não apresentando compromissos conjugais; (d) estar trabalhando ou ter experiência de trabalho. A Tabela 1 apresenta a caracterização dos participantes quanto ao sexo, idade, diagnóstico, nível de escolaridade e status empregatício. Para a identificação dos participantes foi utilizada a letra $\mathrm{P}$ seguida por um número.

Tabela 1

Identificação dos Participantes com DI por Sexo, Idade, Diagnóstico, Nível de Escolaridade e Status Empregatício

\begin{tabular}{cccccc}
\hline Participantes & Sexo & $\begin{array}{c}\text { Idade } \\
\text { (em anos) }\end{array}$ & Diagnóstico $^{\mathrm{a}}$ & Escolaridade & $\begin{array}{c}\text { Status } \\
\text { empregatício }\end{array}$ \\
\hline P1 & Masculino & 23 & Retardo Mental & Sem escolaridade & Empregado \\
P2 & Feminino & 26 & Síndrome de Down & Ensino Médio Completo & Empregado \\
P3 & Masculino & 29 & Retardo Mental & Sem escolaridade & Empregado \\
P4 & Feminino & 40 & Síndrome de Down & Sem escolaridade & Empregado \\
P5 & Masculino & 30 & Retardo Mental Leve & Fundamental Incompleto & Desempregado \\
P6 & Feminino & 36 & Retardo Mental Leve & Ensino Médio Completo & Desempregado \\
P7 & Masculino & 33 & Retardo Mental Grave & Sem escolaridade & Desempregado \\
P8 & Feminino & 28 & Síndrome de Down & Sem escolaridade & Desempregado \\
P9 & Masculino & 25 & Retardo Mental Leve & Fundamental Incompleto & Desempregado \\
\hline
\end{tabular}

a Foram preservados os termos médicos descritos no diagnóstico dos participantes. Portanto, quando se lê a denominação "retardo mental" a mesma refere-se ao atual termo "deficiência intelectual".

Cinco participantes estavam desempregados e quatro empregados, por ocasião da coleta de dados, sendo quatro do sexo feminino e cinco do sexo masculino e havendo pessoas que não frequentaram a escola formal, tendo sido catego- rizados como sem escolaridade $(n=5)$ e pessoas com o ensino médio concluído $(n=2)$.

A Tabela 2 demonstra dados referentes à situação ocupacional dos participantes, destacando-se a função exercida, local e tempo de 
trabalho, carga horária diária e jornada de trabalho. Identifica-se uma variedade nas funções exercidas, havendo mais pessoas empregadas como auxiliares $(n=5)$. Verifica-se uma média de 40,2 meses de tempo de trabalho no grupo das pessoas que estavam empregadas (P1, P2, P3 e
P4) e 15,2 meses no grupo de pessoas desempregadas. A carga horária diária e a jornada de trabalho foram variáveis, tendo pessoas que trabalhavam quatro horas $(n=3)$, seis horas $(n=3)$ e até oito horas $(n=2)$, com jornada de segunda à sexta $(n=4)$, segunda a domingo $(n=2)$ e por escala $(n=3)$.

Tabela 2

Características Ocupacionais dos Participantes com DI por Função Exercida, Local de Trabalho, Tempo de Trabalho, Carga Horária Diária e Jornada de Trabalho

\begin{tabular}{lccccc}
\hline Participantes & $\begin{array}{c}\text { Função que exerce/ } \\
\text { exercia }\end{array}$ & Local de trabalho & $\begin{array}{c}\text { Tempo de } \\
\text { trabalho } \\
\text { (em meses) }\end{array}$ & $\begin{array}{c}\text { Carga } \\
\text { horária } \\
\text { diária }{ }^{\text {a }}\end{array}$ & $\begin{array}{c}\text { Jornada } \\
\text { de trabalho }\end{array}$ \\
\hline P1 & $\begin{array}{c}\text { Empacotador } \\
\text { Digitadora }\end{array}$ & Supermercado & 48 & 4 & Escala \\
P2 & Andústria de Metais & 6 & 6 & Segunda à Sexta \\
P3 & $\begin{array}{c}\text { Auxiliar de serviços } \\
\text { gerais }\end{array}$ & Lanchonete & 48 & 6 & Escala \\
P4 & Auxiliar de serviços & Lanchonete & 60 & 6 & Escala \\
P5 & Empregada doméstica & Residência & 48 & - & Segunda a domingo \\
P6 & Auxiliar de escritório & Indústria Siderúrgica & 6 & 8 & Segunda à Sexta \\
P7 & Secretária & Indústria Siderúrgica & 9 & 4 & Segunda à Sexta \\
P8 & Auxiliar de açougue & Supermercado & 4 & 4 & Segunda à Sexta \\
P9 & Indústria Gráfica & 8 & Segunda a domingo \\
\hline
\end{tabular}

${ }^{\mathrm{a}} \mathrm{O}$ participante $\mathrm{P} 5$ não relatou a carga diária que trabalhava.

\section{Instrumentos}

Foi utilizado o Questionário de Caracterização do Sistema Familiar (Dessen \& Cerqueira-Silva, 2009), o qual teve como finalidade obter uma caracterização demográfica das famílias, contendo itens referentes aos genitores e à família em geral, além de questões sobre habilidades, diagnóstico e situação de trabalho dos participantes com DI. Foram construídos dois roteiros de entrevista semiestruturadas com base na proposta de Lobato (2009) e Masson (2009), sendo um destinado às pessoas com DI que estavam trabalhando e outro às que estavam desempregados. As entrevistas tiveram o objetivo de descrever o processo de profissionalização e escolarização, as expectativas sobre a sua inclusão no trabalho, bem como os sentimentos e reações em relação à sua experiência de trabalho e ao apoio recebido da família.

\section{Procedimentos de Coleta de Dados}

Os participantes foram recrutados a partir do cadastro em um programa de balcão de empregos de uma instituição de assessoria à pessoa com deficiência em uma cidade do interior de Minas Gerais, bem como por meio do contato com uma instituição de atendimento especializado da mesma cidade. De acordo com os critérios, quais sejam: (a) apresentar diagnóstico de DI, sem comorbidades, tendo sido emitido por um médico ou através de relatório psicossocial/psicopedagógico; (b) possuir idade mínima de 18 anos; (c) residir com os genitores ou responsáveis, não apresentando compromissos conjugais; (d) estar trabalhando ou ter experiência de trabalho; disponibilidade e consentimento das instituições foram selecionados nove participantes com DI. Os mesmos foram contatados pelas pesquisadoras, por telefone ou 
pessoalmente, com o propósito de esclarecer os objetivos da pesquisa e solicitar, de forma oral, o consentimento para a participação na investigação. A visita à residência era marcada de acordo com os dias e horários disponibilizados pelo participante com DI. Nesta visita foi assinado o Termo de Consentimento Livre e Esclarecido, aplicado o questionário e realizada a entrevista individual que foi gravada em aparelho de áudio digital e, posteriormente, transcrita na íntegra para análise de conteúdo.

\section{Procedimentos de Análise de Dados}

Para a análise dos resultados do Questionário empregou-se estatística descritiva e para as entrevistas, a análise foi realizada com base na proposta de Dessen e Cerqueira-Silva (2009). Este modelo pressupõe: (a) seleção e exploração do material, denominada pré-análise; (b) codificação; (c) agrupamento dos temas; (d) formação das categorias síntese; (e) classificação dos temas; (f) definição das categorias. Complementarmente, as autoras propõem: (g) revisão do sistema preliminar e elaboração do sistema integrado (definitivo) de categorias e (h) validação do sistema integrado de categorias, a partir da análise de juízes.

\section{Procedimentos Éticos}

A pesquisa foi aprovada pelo Comitê de Ética em Pesquisa Humana da Universidade Federal de Juiz de Fora, sob o sob n ${ }^{\circ} 118 / 2011$, tendo os participantes assinado o Termo de Consentimento Livre e Esclarecido (TCLE).

\section{Resultados}

Os resultados são apresentados, primeiramente, considerando as categorias comuns identificadas tanto nos relatos dos participantes que trabalhavam como naqueles desempregados. Também são descritas as categorias identificadas, separadamente, nas entrevistas com os participantes empregados e com aqueles desempregados.

\section{Período de Escolarização}

Do total de nove participantes, cinco falaram de sua escolarização restringindo-se à descrição das escolas que frequentaram e três mencionaram informações consideradas vagas e imprecisas sobre algum momento ou acontecimento da sua vida escolar, não respondendo à questão propriamente. Apenas um participante afirmou não se lembrar do seu período escolar, não mencionando, portanto, informações a esse respeito. Evidenciou-se, pelos relatos, a dificuldade dos participantes em descrever a respeito desse período de suas vidas, uma vez que havia a necessidade de que eles se lembrassem de um período remoto.

\section{O Significado de Trabalhar}

Os relatos dos participantes indicaram que 'estar trabalhando' significa: (a) adquirir melhorias financeiras $(n=5)$ e (b) alcançar melhorias pessoais $(n=4)$. Um participante não relatou sobre o significado de estar trabalhando. As melhorias financeiras relatadas se referiram à: independência financeira $(n=2)$, sustento do lar/casa/ vida $(n=2)$ e aquisição de bens e lazer $(n=1)$. Já as melhorias pessoais foram satisfação pessoal $(n=2)$, produtividade $(n=1)$ e responsabilidade $(n=1)$, conforme indicam os relatos:

Melhorias financeiras:

Independência financeira: "Bom... eu tenho o meu dinheiro..." (P1).

Sustento do lar/casa/vida: "Trabalhar é melhorar a situação aqui dentro da minha casa" (P8).

Aquisição de bens e lazer: “. . . vocêter o seu dinheiro, comprar roupa, seu tênis e passear" (P9).

Melhorias Pessoais:

Satisfação pessoal: "Ah, que significa, porque eu me sinto bem" (P5).

Produtividade: "Estarei sendo produtiva, produzir" (P6).

Responsabilidade: "Trabalhar significa pra mim é ter hora marcada, é acordar cedo... $\mathrm{Na}$ verdade eu sou responsável, por dormir cedo, na hora certa, me apronto cedo e vou trabalhar" (P2). 
No tocante ao surgimento do interesse em trabalhar, os relatos evidenciaram que este foi devido: (a) à motivação para comprar, através do salário proporcionado pelo emprego $(n=1)$; (b) aos incentivos recebidos pela família $(n=1)$ e (c) à mediação da instituição de atendimento especializado que frequentavam $(n=2)$. Houve relatos vagos e imprecisos $(n=4)$ e um participante não soube responder à pergunta. Abaixo se encontram exemplos dos relatos de acordo com as categorias apresentadas:

Motivação para comprar: "Meu interesse em trabalhar é mais para comprar as coisas para mim que eu preciso, né..." (P5).

Incentivo de familiares: "Assim, minha mãe falava 'tem que trabalhar' e fui procurando, procurando..." (P6).

Intermédio de instituições: "O meu chefe, chamado $C$., . . ele foi lá na $X$ [instituição especializada] e me trouxe para trabalhar aqui no $Z$ [empresa contratante] . . . Então pela $X$ [instituição especializada] que eu fui encaminhado pra lá" (P3).

Em relação à satisfação com a inclusão no trabalho, os participantes relataram que este processo foi satisfatório $(n=4)$, insatisfatório $(n=$ $2)$ e ainda um direito $(n=1)$. Um participante relatou não saber falar sobre o assunto e outro relatou informações vagas e imprecisas. Os relatos abaixo exemplificam as categorias encontradas:

Satisfatório: "Eu acho que é muito bom, sabe? ... eu acho que ela [pessoa com deficiência] ganha potencial pra trabalhar...” (P2). "Ah é bom, né..." (P5).

Insatisfatório: "São muito desvalorizados, porque as pessoas que são mais do que você acham que são melhores do que você e todo mundo está na mesma faixa, ganhando a mesma coisa..." (P3).

Direito: “. . . eu não quero dizer que as pessoas são melhores que a gente, tudo na vida tem lei e ela deve ser cumprida" (P8).

\section{Percepção sobre o Ambiente de Trabalho e Relações Interpessoais}

Foram investigadas as condições do ambiente de trabalho tanto dos participantes empregados quanto dos desempregados, tendo sido proposto a eles que descrevessem o ambiente físico, constituído por aspectos como o mobiliário e equipamentos utilizados, e o ambiente social, isto é, as pessoas, as relações com colegas de trabalho e empregadores. Tanto o ambiente físico como o social foram descritos como satisfatórios na maior parte dos relatos $(n=7)$. Identificou-se um relato em que o ambiente social foi descrito como inicialmente satisfatório e outro com informações imprecisas ou vagas. Seguem-se exemplos dos relatos de acordo com as categorias:

Ambientes físico e social satisfatórios: " $\mathrm{La}$ é um lugar tranquilo... as pessoas são boas, me ajudavam" (P6). "Assim... . . . eu gosto muito, eles gostam de mim, ganho presente de clientes, caixa de bombom... Tem luvas, tem luvas para lavar banheiro, tudo direitinho..." (P4). "O local é legal, eu gostei muito de ficar lá... minha sala é individual, tinha crachá e nome na porta... eles fizeram festa de aniversário pra mim" (P2).

Ambiente social inicialmente satisfatório: "O local era até bom, os colegas, mas depois foi gerando uma fofocaiada... todo serviço tem fofoca né" (P9).

Não explicita: "Tinha uma van que levava e busca a gente lá... a van pegava a gente no setor lá e levava a gente pro refeitório no almoço, por volta de onze, onze e meia" (P7).

\section{O Papel da Família na Inclusão}

Com relação ao papel da família no processo de inclusão no mercado de trabalho, houve relatos evidenciando que esta teve a função de apoiar e ajudar $(n=6)$, entretanto, um relato indicou o não envolvimento da família nesse processo e dois participantes não relataram. Abaixo se apresentam os relatos:

Apoiar/Ajudar: "Os meus pais acham muito bom eu trabalhar, eu trabalhar lá... Eles me apoiam, me ajudam, me tratam bem, sabe?..." (P2).

A minha mãe me ajudou, me deu todo apoio, porque tem mãe que não, ah o filho é bobo, mas com medo das pessoas enganar, tudo isso... E agora que eu estou trabalhando eles continua me apoiando do mesmo jeito. (P3)

Não envolvimento: "Aí minha mãe diz pra mim que eu tenho que trabalhar, então eu falo... 
'então arruma um pra mim'... E o meu pai me cobrando..." (P6).

\section{Realizações com o Trabalho}

As perguntas referentes a essa categoria foram realizadas apenas aos participantes empregados. No que se refere às realizações que $o$ trabalho proporciona aos participantes empregados, foram identificados: desenvolvimento social $(n=2)$, amadurecimento pessoal $(n=1)$ e reconhecimento social $(n=1)$. Exemplos de relatos são descritos abaixo:

Desenvolvimento social: "Ah, para mim muita coisa vem acrescentando... eu comecei a lidar com todo mundo, conhecer pessoas..." (P4).

Amadurecimento pessoal: "Eu aprendi com meus amigos, eu amadureci, cresci lá..." (P2).

Reconhecimento social: "Eu comecei a ter o respeito mais das pessoas, valorizou bastante..." (P3).

Em relação ao salário recebido, os participantes indicaram que o utilizam para aquisição de bens materiais, conforme se constatam pelos relatos:

"Comprar roupa" (P1). "Eu comprei um rádio pra mim, no carnaval... e está sendo assim, compra tênis de vez em quando, uma roupa, quando não der eu não compro...” (P3). “. . . eu compro roupa, tênis, eu já comprei dois tênis, um para trabalhar e um pra sair..." (P4).

\section{Expectativas em Relação ao Emprego e Motivos do Desligamento Ocupacional}

No que se refere às expectativas em relação ao trabalho, pergunta realizada apenas aos participantes desempregados, estes relataram: melhorias financeiras $(n=3)$, valorização das habilidades $(n=1)$ e satisfação pessoal $(n=1)$. Houve ainda um relato com informações vagas e imprecisas. Exemplos de relatos são apresentados:

Melhorias financeiras: "Depois que eu começar a trabalhar é melhorar minha vida, ajudar meu pai e minha mãe..." (P9). "Pode mudar mais a minha situação né...” (P7).

Valorização das habilidades: "Ser uma bailarina de sucesso e ser reconhecida lá fora.
Para todo mundo ver que sou muito capaz de fazer" (P8).

Satisfação pessoal: "Tudo né... minha auto estima levanta de novo..." (P6).

Considerando que todos os participantes desempregados já tiveram experiências de trabalho, os mesmos relataram como motivo de saída do último emprego: o cumprimento ou não das normas de trabalho $(n=3)$, dificuldades em aprender tarefas $(n=1)$ e inadequação ao cargo $(n=1)$, conforme mostram os relatos:

Cumprimento ou não das normas de traba1ho: "Acabou o contrato, mas eu não queria, não queria mesmo" (P8). "Mas, o pessoal não queria pagar... aí.. para evitar problemas maiores, eu sai fora" (P5).

Dificuldades em aprender tarefas: "Era dificil pra mim entender as coisas lá... tinha que cortar e separar as coisas lá, tinha que usar uma luva pesada na mão esquerda..." (P9).

Inadequação ao cargo: "Eu tinha que ficar até tarde da noite... e a moça achava que eu era lenta demais" (P6).

\section{Discussão}

Investigar e pesquisar as pessoas com DI traduz em desafio para o pesquisador, mas os resultados da presente investigação mostraram a viabilidade da coleta de dados junto a essas pessoas. Houve relatos imprecisos e também a dificuldade em descrever sobre algum assunto proposto, mesmo assim, os objetivos foram alcançados de forma adequada. O roteiro de entrevista utilizado se mostrou um instrumento bastante eficaz para a coleta de dados, principalmente por permitir investigar, por meio dos relatos orais, o fenômeno da inclusão no trabalho (Lobato, 2009). O roteiro de entrevista com as pessoas com DI foi construída considerando o nível de compreensão dos adultos participantes, proporcionando dar "voz" a estas pessoas. Apesar do cuidado na construção do roteiro de entrevista, houve algumas questões que alguns participantes deixaram de responder, provavelmente por não compreendê-las. Assim, ressalta-se a necessidade de maior adequação dos instrumentos de coleta de dados às características da 
pessoa. Destaca-se que este procedimento pode constituir-se um desafio aos pesquisadores, porém, a realização de estudos pilotos pode minimizar este tipo de situação.

No geral, os resultados obtidos no presente trabalho corroboram os dados da literatura que denotam a importância da inclusão no mercado de trabalho para o desenvolvimento das pessoas adultas com DI e sinalizam para a necessidade de aprimoramento desse processo (Akkerman et al., 2014; Araújo \& Schmidt, 2006; Holwerda et al., 2013; Lobato, 2009; Masson, 2009; Pereira-Silva $\&$ Furtado, 2012; Santilli et al., 2014; Tanaka \& Manzini, 2005). Pode-se afirmar que os participantes com DI percebem a inclusão no mercado de trabalho como um processo satisfatório, mas também insatisfatório, sendo um direito e um meio de capacitação das habilidades. Segundo os participantes o trabalho lhes proporciona tanto melhorias financeiras como pessoais, tais como desenvolvimento social, amadurecimento, o sentimento de ser produtivo e também o reconhecimento social, conforme resultados também encontrados por outros estudos (Akkerman et al., 2014; Santilli et al., 2014).

A literatura aponta a escolaridade e a preparação profissional como requisitos importantes para se inserir no emprego (Lobato, 2009; Masson, 2009; Nord et al., 2013). No entanto, no presente estudo, cinco participantes não possuíam nível de escolaridade, embora tenham participado de Oficinas profissionalizantes em instituições de atendimento especializado, sendo que três desses participantes estavam empregados por ocasião da coleta de dados. Esse resultado parece indicar que a falta de escolarização, por si só, não tem se traduzido em barreira para a inclusão no trabalho, não sendo, portanto, um critério exclusivo para a contratação da pessoa com DI. A literatura ressalta que a falta de acessibilidade social nas empresas contratantes e a falta de formação profissional das pessoas com DI são as grandes dificuldades na inclusão dessas pessoas no mercado de trabalho (Araújo \& Schmidt, 2006; Lobato, 2009; Masson, 2009; Neves-Silva, Prais, \& Silveira, 2015; Nord et al., 2013; Paiva et al., 2015; Pereira-Silva \& Furtado, 2012). Vale ressaltar que a participante que possuía maior nível de escolaridade (P2), ensino médio completo, estava empregada com o cargo de digitadora/auxiliar de escritório em uma empresa de metais. Essa participante também frequentou Oficinas desde criança. Esse resultado reforça a importância da escolaridade e da preparação profissional para melhor inserção no mercado. Mas também há necessidade de treinamento e preparação, uma vez que havia, dentre os participantes, um desempregado com o ensino médio completo. Destaca-se que não houve relatos de descontentamento com a inclusão, porém, ainda há falta de oportunidades, de profissionalização, de vagas de emprego e de valorização da pessoa com deficiência intelectual, conforme destacado por Neves-Silva et al. (2015).

Os relatos sobre 'o que significa estar trabalhando' evidenciaram que os participantes têm clareza sobre o que o status ocupacional pode lhes proporcionar, especialmente no tocante à autonomia, independência, satisfação pessoal, produtividade e responsabilidade, indicando que o ambiente de trabalho pode favorecer avanços em aspectos do desenvolvimento, o que é coerente com o modelo bioecológico de Bronfenbrenner (Bronfenbrenner \& Morris, 1998). No tocante ao desenvolvimento pessoal, Akkerman et al. (2014) também identificaram em seu estudo a importância atribuída ao trabalho, seja no desenvolvimento de vínculos sociais, de habilidades e competências ou de autonomia.

Vale destacar a importância das empresas contratantes em estabelecer um ambiente promotor de desenvolvimento e de atitudes inclusivas (Gomes-Machado \& Chiari, 2009; Nota et al., 2014) pois, muitas vezes, a dificuldade não se encontra apenas na inserção da pessoa com DI no emprego, mas na sua permanência (Cook, Foley, \& Semeah, 2016), principalmente pela falta de acessibilidade social nas empresas (Cook et al., 2016; Lobato, 2009; Masson, 2009). Não houve muitos relatos sobre dificuldades no processo de inclusão dos participantes na presente investigação, apesar de alguns consideraram a inclusão insatisfatória sendo também o ambiente considerado não totalmente adequado. Assim, conforme destacado por Nota et al. (2014), há necessidade da implementação de intervenções 
dentro das empresas que visem eliminar o preconceito de empregadores no tocante à contratação de pessoas com deficiência, principalmente a intelectual.

Em relação ao papel da família no processo de inclusão no trabalho, a maior parte dos participantes apontou a família como exercendo a função de apoiar, oferecendo suporte emocional, incentivando e auxiliando-os ao longo de seu processo de inclusão. Por outro lado, houve um relato de não envolvimento da família com o processo de inclusão. Dessa forma, pode-se considerar que a maioria das famílias mostrou-se envolvida, de alguma forma, na inclusão de seu membro com DI. A importância da família para os adultos com DI e a influência desta no processo de inclusão ainda carece de estudos, porém, a família pode ser compreendida como essencial para que ocorra a inclusão da pessoa com DI (Furtado \& Pereira-Silva, 2016; Pereira-Silva \& Furtado, 2012), conforme apontam os dados da presente investigação. Sugere-se a implementação de estudos que visem identificar as possíveis relações entre a efetividade da inclusão e o comprometimento da família, subsidiando intervenções que objetivem desenvolver ações dirigidas às famílias para a promoção da inclusão no trabalho.

\section{Considerações Finais}

É fundamental que família, comunidade, instituições de atendimento especializado, escolas e empresas formem redes de apoio que são essenciais ao desenvolvimento das pessoas com DI (Dallabrida, 2007; Neves-Silva et al., 2015; Rosa \& Denari, 2013). Esses diferentes ambientes podem ser considerados como contextos de desenvolvimento e inclusão social. Portanto, destaca-se a importância do papel representado pelos mesmos e principalmente da família como mediadora das relações entre as pessoas com DI e os outros contextos, como por exemplo, o ambiente de trabalho (Bronfenbrenner \& Morris, 1998). O contexto do trabalho pode ser um grande promotor de desenvolvimento para as pessoas com DI (Akkerman et al., 2014; Santilli et al., 2014) e os dados da presente investigação apontam para essa direção, especialmente, quando associam-se os relatos de melhorias pessoais e satisfação com o processo de inclusão no trabalho.

É importante ressaltar que os dados desta investigação foram baseados em relatos de uma reduzida amostra de adultos com DI de uma cidade do interior de Minas Gerais, o que traz algumas limitações concernentes às considerações aqui realizadas. Destaca-se a importância de implementar estudos que visam compreender melhor o processo de inclusão, bem como de agregar mais eficácia a esse processo. Para tal propósito, sugere-se que investigações futuras busquem uma análise sistêmica, com coletas a partir de diversificados participantes, tais como os familiares, os empregadores, os colegas de trabalho e os profissionais das instituições de atendimento especializado. A implementação de estudos que visem um melhor entendimento do processo de inclusão no mercado de trabalho, bem como seu impacto no desenvolvimento das pessoas com DI, torna-se de grande relevância para a compreensão do desenvolvimento humano e para a implementação e planejamento de políticas públicas de inclusão.

\section{Referências}

Akkerman, A., Janssen, C. G. C., Kef, S., \& Meininger, H. P. (2014). Perspectives of employees with intellectual disabilities on themes relevant to their job satisfaction: An explorative study using photovoice. Journal of Applied Research in Intellectual Disabilities, 27, 542-554. doi: 10.1111/jar.12092

Almeida, L. A. D., Carvalho-Freitas, M. N., \& Marques, A. L. (2010). Inserção no mercado formal de trabalho: Satisfação e condições de trabalho sob o olhar das pessoas com deficiência. In M. N. Carvalho-Freitas \& A. L. Marques (Eds.), O trabalho e as pessoas com deficiência: Pesquisas, práticas e instrumentos de diagnóstico (pp. 89-105). Curitiba, PR: Juruá.

Aranha, M. S. F. (2001). Paradigmas da relação da sociedade com as pessoas com deficiência. Revista do Ministério Público do Trabalho, 21, 160-173. Recuperado em http://www.anpt.org. br/index2.jsp?tipo_noticia $=7 \&$ pagina $=3$ 
Araújo, E. A. C., Escobal, G., \& Ribeiro, D. M. (2006). Planejamento e organização de serviços para a formação de pessoas com deficiência mental para o trabalho. In C. Goyos \& E. Araújo (Eds.), Inclusão social: Formação do deficiente mental para o trabalho (pp. 143-166). São Carlos, SP: RiMa.

Araújo, J. P., \& Schmidt, A. (2006). A inclusão de pessoas com necessidades especiais no trabalho: A visão de empresas e de instituições educacionais especiais na cidade de Curitiba. Revista Brasileira de Educação Especial, 12, 241-254. doi: 10.1590/S1413-65382006000200007

Barbosa-Gomes, J. F., \& Carvalho, M. O. (2010). $\mathrm{O}$ profissional com deficiência e o mercado de trabalho: Parceria de sucesso. In M. N. Carvalho-Freitas \& A. L. Marques (Eds.), Trabalho e pessoas com deficiência: Pesquisa, práticas e instrumentos de diagnóstico (pp. 201-212). Curitiba, PR: Juruá.

Bronfenbrenner, U., \& Morris, P. A. (1998). The ecology of developmental processes. In W. Damon \& R. M. Lerner (Eds.), Handbook of child psychology: Theoretical models of human development (pp. 993-1028). New York: Wiley.

Cezar, K. R. (2010). As pessoas com deficiência intelectual e o direito à inclusão no trabalho: $A$ efetividade da lei de cotas (Dissertação de mestrado, Universidade de São Paulo, SP, Brasil).

Constituição da República Federativa do Brasil. (1988). Promulgada em 5 de outubro de 1988 e atualizada até a Emenda Constitucional $n^{\circ} 88$, de 15 de setembro de 2015. Recuperado em http:// www.planalto.gov.br/ccivil_03/Constituicao/ Constituicao.htm

Cook, L. H., Foley, J. T., \& Semeah, L. M. (2016). An exploratory study of inclusive worksite wellness: Considering employees with disabilities. Disability and Health Journal, 9, 100-107. doi: 10.1016/j.dhjo.2015.08.011

Dallabrida, A. M. (2007). As famílias e a classe especial em um colégio de elite. Revista Brasileira de Educação Especial, 13, 459-478. doi: 10.1590/S1413-65382007000300011

Dessen, M. A., \& Cerqueira-Silva, S. (2009). Desenvolvendo sistemas de categorias com dados de entrevistas. In L. Weber \& M. A. Dessen (Eds.), Pesquisando a família: Instrumentos para coleta e análise de dados (pp. 43-56). Curitiba, PR: Juruá.
Furtado, A. V., \& Pereira-Silva, N. L. P. (2014). Trabalho e pessoas com deficiência intelectual: Análise da produção científica. Revista Brasileira de Orientação Vocacional, 15(2), 213-223. doi: http://pepsic.bvsalud.org/scielo.php?script $=$ sci arttext\&pid=S1679-33902014000200011

Furtado, A. V., \& Pereira-Silva, N. L. (2016). Deficiência intelectual e trabalho: A inclusão segundo os membros familiares. In N. L. Pereira-Silva, A. G. Barbosa, \& M. C. Rodrigues (Eds.), Pesquisas em desenvolvimento humano e educação (pp. 273-296). Curitiba, PR: CRV.

Gold, P. B., Fabian, E. S., \& Luecking, R. G. (2013). Job acquisition by urban youth with disabilities transitioning from school to work. Reabilitation Cousenling Bulletin, 57(1), 31-45. doi: $10.1177 / 0034355213481248$

Gomes-Machado, M. L., \& Chiari, M. B. (2009). Estudo das habilidades adaptativas desenvolvidas por jovens com síndrome de Down incluídos e não incluídos no mercado de trabalho. Saúde $e$ Sociedade, 18, 652-661. doi: 10.1590/S010412902009000400009

Holwerda, A., van der Klink, J. J. L., de Boer, M. R., Groothoff, J. W., \& Brouwer, S. (2013). Predictors of work participation of young adults with mild intellectual disabilities. Research in Developmental Disabilities, 34, 1982-1990. doi: 10.1016/j.ridd.2013.03.018

Instituto Brasileiro de Geografia e Estatística. (2012). Censo Demográfico 2010: Características gerais da população, religião e pessoas com deficiência. Recuperado em http://www.ibge.gov. br/home/estatistica/populacao/censo2010/caracteristicas_religiao_deficiencia/default_caracteristicas_religiao_deficiencia.shtm

Kirsh, B., Stergiou-Kitab, M., Gewurtz, R., Dawson, D., Krupa, T., Lysaght, R., \& Shaw, L. (2009). From margins to mainstream: What do we know about work-integration for persons with brain injury, mental illness and intellectual disability? Work, 32, 391-405. doi: 10.3233/WOR-20090851

Lei Federal $n^{\circ} 8.112$ de 1990. (1990). Dispõe sobre o regime jurídico dos servidores públicos civis da União, das autarquias e das fundações públicas federais. Recuperado em http://www.planalto. gov.br/ccivil_03/leis/L8112cons.htm

Lei $n^{\circ}$ 8.213. (1991). Dispõe sobre os Planos de Benefícios da Previdência Social e dá outras provi- 
dências. Diário Oficial da República Federativa do Brasil. Recuperado em http://www.planalto. gov.br/ccivil_03/leis/18213cons.htm

Lei 13.146 de julho de 2015. (2015). Institui a Lei Brasileira de Inclusão da Pessoa com Deficiência (Estatuto da Pessoa com Deficiência). Recuperado em http://www.planalto.gov.br/ccivil_03/_ato2015-2018/2015/lei/113146.htm

Lobato, B. C. (2009). Pessoas com deficiência no mercado de trabalho: Implicações da lei de cotas (Dissertação de mestrado não-publicada, Centro de Educação e Ciências Humanas, Universidade Federal de São Carlos, SP, Brasil).

Lysaght, R., Cobigo, V., \& Hamilton, K. (2012). Inclusion as a focus of employment-related research in intelectual disabity from 2000 to 2010: A scoping review. Disability \& Rehabilitation, 34(16), 1339-1350. doi: $10.3109 / 09638288.2011 .644023$

Lysaght, R., Petner-Arrey, J., Cobigo, V., \& OulletteKuntz, H. (2014). Work preparation and participation in Ontario for persons with intellectual and development disabilities - A cross-region analysis. Journal on Developmental Disabilities, 20(2), 34-43. Retrieved from http://www. oadd.org/docs/41018_JoDD_20-2_v10f_34-43 lysaght_et_al.pdf

Mantoan, M. T. E. (2015). Inclusão escolar: O que é? Por quê? Como fazer? São Paulo, SP: Summus.

Masson, M. J. B. (2009). Educação e trabalho: A constituição do trabalhador deficiente intelectual (Dissertação de mestrado não-publicada, Universidade Metodista de Piracicaba, Piracicaba, SP, Brasil).

Mendes, E. G. (2006). A radicalização do debate sobre inclusão escolar no Brasil. Revista Brasileira de Educação, 11(33), 387-405.

Morin, D., Rivard, M., Crocker, A. G., Boursier, C. P., \& Caron, J. (2013). Public attitudes towards intellectual disability: A multidimensional perspective. Journal of Intellectual Disability Research, 53(3), 279-292. doi: 10.1111/ jir.12008

Neves-Silva, P., Prais, F. G., \& Silveira, A. M. (2015). Inclusão da pessoa com deficiência no mercado de trabalho em Belo Horizonte, Brasil: Cenário e perspectiva. Ciência \& Saúde Coletiva, 20(8), 2549-2558. doi: 0.1590/141381232015208.17802014
Nord, D., Luecking, R., Mank, D., Kiernan, W., \& Wray, C. (2013). The state of the science of employment and economic self-sufficiency for people with intellectual and developmental disabilities. Intellectual and Developmental Disabilities, 51(5), 376-384. doi: 10.1352/19349556-51.5.376

Nota, L., Santilli, S., Ginevra, M. C., \& Soresi, S. (2014). Employer attitudes towards the work inclusion of people with disability. Journal of Applied Research in Intellectual Disabilities, 27, 511-520. doi: 10.1111/jar.12081

Organização das Nações Unidas. (1948). Resolução 217, de 10 de dezembro de 1948. Declaração Universal dos Direitos Humanos. Recuperado em http://www.dudh.org.br/wp-content/uploads/2014/12/dudh.pdf

Paiva, J. C. M., Bendassolli, P. F., \& Torres, C. C. (2015). Sentidos e significados do trabalho: Dos impedimentos às possibilidades do trabalho de pessoas com deficiências. Estudos e Pesquisas em Psicologia, 15(1), 218-239. Recuperado em http://www.e-publicacoes.uerj.br/index.php/revispsi/article/view/16071/12091

Pereira-Silva, N. L., \& Furtado, A. V. (2012). Inclusão no trabalho: A vivência de pessoas com deficiência intelectual. Interação em Psicologia, 16(1), 95-100. doi: http://dx.doi.org/10.5380/ psi.v16i1.23012

Rosa, F. D., \& Denari, F. E. (2013). Trabalho, educação e família: Perspectivas para a pessoa com deficiência intelectual. Revista Educação Especial, 26(45), 73-90. Recuperado em http://www. redalyc.org/articulo.oa? $\mathrm{id}=313128573006$

Santilli, S., Nota, L., Ginevra, M. C., \& Soresi, S. (2014). Career adaptability, hope and life satisfaction in workers with intellectual disability. Journal of Vocational Behavior, 84(1), 21-30. doi: 10.1016/j.jvb.2014.02.011

Sassaki, R. K. (1997). Inclusão. Construindo uma sociedade para todos (3. ed.). Rio de Janeiro, RJ: WVA.

Tanaka, E. D. O., \& Manzini, E. J. (2005). O que os empregadores pensam sobre o trabalho da pessoa com deficiência? Revista Brasileira de Educação Especial, 11, 273-294. doi: 10.1590/ S1413-65382005000200008

Vieira, C. M., \& Denari, F. E. (2008). Concepções infantis sobre a deficiência mental: Efeitos de um 
programa informativo. In M. A. Almeida, E. G. Mendes, \& M. C. P. I. Hayashi (Eds.), Temas em educação especial: Deficiências sensoriais e deficiência mental (pp. 289-297). Brasília, DF: PROESP.

Vornholt, K., Uitdewilligen, S., \& Nijhuis, F. J. N. (2013). Factors affecting the acceptance of people with disabilities at work: A literature review.
Journal Occupational Reahabilitation, 23(4), 463-473. doi: 10.1007/s10926-013-9426-0

Recebido: 05/08/2016

$1^{a}$ revisão: $30 / 06 / 2017$

Aceite final: 16/07/2017 